Editorial

\title{
The Continuum of Parainfectious Reactions of the Cerebellum in Childhood*
}

\author{
Maja Steinlin ${ }^{1}$ \\ ${ }^{1}$ Department of Neuropaediatrics, University Children's Hospital, \\ Bern, Switzerland
}

Neuropediatrics 2012;43:238-239.

Acute cerebellitis is thought to be a rare problem in childhood; however, its more benign variant-acute cerebellar ataxia-is the most frequent reason for children presenting in an emergency department for ataxia. ${ }^{1}$ With increasing use of magnetic resonance imaging in children with acute ataxia, it becomes more and more obvious that there is a continuum of parainfectious reactions of the cerebellum in childhood. It spans from the most benign form of acute ataxia to acute cerebellitis and to the most severe form of cerebellar swelling with hydrocephalus and tonsillar herniation. ${ }^{2-4}$

Acute ataxia is characterized by an acute onset of ataxia in an otherwise healthy child. ${ }^{5,6}$ Magnetic resonance imaging is usually normal and cerebrospinal fluid (CSF) might show some cells and elevated protein content as signs of an inflammatory reaction. In contrary, children with acute cerebellitis are more affected. ${ }^{2,3,7}$ Typically, the onset of ataxia is preceded or accompanied by more generalized symptoms such as headache or a change of mental state, as is shown in more than half of the children. The more pronounced clinical problems are underlined by additional magnetic resonance abnormalities as patchy demyelinization of the cerebellum or diffuse T2 hyperintensity or even swelling. ${ }^{8}$ CSF is similar to acute ataxia with some mild inflammatory signs. However, lumbar puncture has to be avoided in most cases, because of the risk of secondary herniation of the tonsils. The most severe form of a parainfectious reaction of the cerebellum is an acute swelling leading to obstruction of the fourth ventricle and resulting in acute hydrocephalus and/or tonsillar herniation, ${ }^{4,9}$ with or without patchy demyelinating or necrotic areas within the cerebellum. These children show severe generalized symptoms with significant changes of mental consciousness, severe ataxia frequently with loss of sitting and walking

\footnotetext{
${ }^{*}$ This article is an editorial on "Long-term outcome of children with acute cerebellitis" by Hennes et al Neuropediatrics 2012; 43(5):240-248.
}

abilities, and in addition, signs and symptoms of impaction of the cerebellum within the posterior fossa. CSF is similar as in acute cerebellitis with pleocytosis as sign of an inflammatory reaction. Histopathology shows patchy necrotic areas in the cerebellum, which leads to marked cerebellar atrophy on follow-up. ${ }^{10}$ If left untreated, this severe form of cerebellitis leads to death.

The mild form of "pure" acute ataxia does not need an immunosuppressive treatment with steroids. Also, treatment with acyclovir in the varicella related cases does not show a better or faster improvement. Despite lack of evidence, in view of the marked symptoms in cerebellitis and especially in fear of progressing of the disease into the most pronounced stage of swelling of the cerebellum, most children with acute cerebellitis are treated with high dose of steroids, which usually leads to a quick and marked improvement of the symptoms. ${ }^{7}$ Children with the most severe form of parainfectious cerebellar affection might need acute shunting of hydrocephalus or decompression of posterior fossa. $^{4,9,10}$

It is well known that children after acute ataxia recover completely from the symptoms of ataxia within a few days or weeks. ${ }^{5,6}$ As the article of Hennes et $\mathrm{al}^{7}$ shows, the group of children with cerebellitis might recover from their neurological symptoms, but almost half of them remain with some mild cerebellar signs as tremor or balance problems. It is easy to understand that the group of children with acute cerebellar swelling show most pronounced long-term problems with mild-to-moderate residual ataxia in almost all cases. $^{4,9,10}$

With increasing awareness of the nonmotor functions of the cerebellum, outcomes of these children are also reported considering their cognitive and behavior problems. Already children with the mildest form of parainfectious cerebellar ataxia show prolonged transient cognitive and behavior problems, which persist in approximately $10 \%$ of children ${ }^{6}$-a prognosis that is frequently neglected in these children. Hennes et $\mathrm{al}^{7}$ show that about $50 \%$ of children with cerebellitis have
Address for correspondence

Prof. Dr. Maja Steinlin, MD, Department of Neuropaediatrics, University Children's Hospital, Inselspital, 3010 Bern, Switzerland (e-mail: maja.steinlin@insel.ch).
Copyright $\odot 2012$ by Thieme Medical Publishers, Inc., 333 Seventh Avenue, New York, NY 10001, USA. Tel: +1(212) 584-4662.
DOI http://dx.doi.org/ 10.1055/s-0032-1327470. ISSN 0174-304X. 
neurocognitive problems-an outcome that seems to go in parallel with the neurological sequelae in these children and is also reflected by cerebellar atrophy observed during followup. ${ }^{8}$ The few case reports with the most pronounced form of acute cerebellar swelling compared with the life-threatening acute stage show astonishingly good outcome. However, they show most pronounced residual symptoms from all groups. Usually there is only mild persistent ataxia but majorly important neurocognitive and behavior problems. ${ }^{4,9,10}$ The degree of cerebellar atrophy in these children is even more pronounced than in those after acute cerebellitis. ${ }^{10}$

In view of the continuum of this clinical picture of parainfectious reaction of the cerebellum in early childhood, it is important to have more detailed knowledge on outcome of these different subgroups. The article of Hennes et al, ${ }^{7}$ for the first time, reporting not only single case reports but also a series of children recovering from acute cerebellitis is of great importance not only to point out the differences of acute cerebellar ataxia to acute cerebellitis but also to underline the differences in outcome prognosis of these two subgroups of children! With lack of evidence for treatment indication, knowledge on outcome of these different groups will help us for our clinical decisions on treatment in the acute phase.

\section{References}

1 Salas AA, Nava A. Acute cerebellar ataxia in childhood: initial approach in the emergency department. Emerg Med J 2010; 27(12):956-957

2 Desai J, Mitchell WG. Acute cerebellar ataxia, acute cerebellitis, and opsoclonus-myoclonus syndrome. J Child Neurol 2012 (e-pub ahead of print). doi: 10.1177/0883073812450318

3 Sawaishi Y, Takada G. Acute cerebellitis. Cerebellum 2002;1(3): 223-228

4 Kamate M, Chetal V, Hattiholi V. Fulminant cerebellitis: a fatal, clinically isolated syndrome. Pediatr Neurol 2009;41:220-222

5 Nussinovitch M, Prais D, Volovitz B, Shapiro R, Amir J. Postinfectious acute cerebellar ataxia in children. Clin Pediatr (Phila) 2003;42(7):581-584

6 Connolly AM, Dodson WE, Prensky AL, Rust RS. Course and outcome of acute cerebellar ataxia. Ann Neurol 1994;35(6):673-679

7 Hennes EM, Zotter S, Dorninger L, et al. Long-term outcome of children with acute cerebellitis. Neuropediatrics 2012;43(5): 240-248

8 Tlili-Graiess K, Mhiri Souei M, Mlaiki B, et al. [Imaging of acute cerebellitis in children. Report of 4 cases]. J Neuroradiol 2006; 33(1):38-44

9 Shkalim V, Amir J, Kornreich L, Scheuerman O, Straussberg R. Acute cerebellitis presenting as tonsillar herniation and hydrocephalus. Pediatr Neurol 2009;41(3):200-203

10 Burri SM, Krauss JK, Schroth G, Weis J, Steinlin M. Near-fatal cerebellar swelling caused by acute multifocal cerebellar necrosis. Eur J Paediatr Neurol 2003;7(3):139-142 